Trauma Berufskrankh $2003 \cdot 5$ [Suppl 2]: S305-S308 DOI 10.1007/s10039-002-0648-1
Septische Chirurgie

M. Schaan ${ }^{1} \cdot$ M. Bierschneider ${ }^{1} \cdot$ A. Kemmer ${ }^{2} \cdot$ H. Jaksche $^{1}$

${ }^{1}$ Neurochirurgie, BG-Unfallklinik Murnau

2Druckkammerzentrum Murnau, BG-Unfallklinik Murnau

\section{Die Behandlung des Hirnabszesses}

\section{Eine interdisziplinäre Aufgabe}

\section{Zusammenfassung}

In den Jahren 1999-2001 wurden 11 Patienten mit Hirnabszessen zuerst operativ, anschließend mit hyperbarer Oxygenierung (HBO) behandelt: 8 Männer und 3 Frauen im Alter von 16-89 Jahre. Davon waren 4 Fälle per continuitatem, 5 hämatogen und 2 iatrogen entstanden. Bei allen Patienten wurden neuronavigations- und ultraschallunterstützt offene Kraniotomien oder Bohrlochkraniotomien durchgeführt. In 8 Fällen konnten Anaerobier isoliert werden, bei 1 Patient Aspergillus, zusätzlich traten 2 iatrogene Infekte mit Staph. aureus auf. Alle Patienten wurden mit mindestens 10 Behandlungssitzungen $\mathrm{HBO}$ (2,4 Bar über $140 \mathrm{~min}$ ) behandelt. Bei einem 46-jährigen Patienten bestand neben der Abszesshöhle eine klinisch inapparente Infarzierung, die weitere Verlaufskontrolle zeigte eine komplette Regression des Infarktes. Ein Patient verstarb. Im weiteren Followup von 8 Monaten traten keine Abszessrezidive auf.Die HBO-Therapie stellt somit eine sinnvolle Ergänzung der Behandlung von Hirnabszessen dar, insbesondere beim Vorliegen anaerober Keime. Eine endgültige Beurteilung dieses Vorgehens kann jedoch erst nach Erfahrungen mit einem größeren $\mathrm{Pa}$ tientenkollektiv erfolgen.

\section{Schlüsselwörter}

Hirnabszess · Kraniotomie - Neuronavigation . Hyperbare Oxygenierung · Aaerobier
D (Abb. 1) stellt hohe Ansprüche an neurochirurgische Fähigkeiten, infektiologische Kenntnisse sowie an die Indikationsstellungen adjuvanter Therapieformen. Neben der klassischen neurochirurgischen Therapie haben sich mittlerweile zusätzliche operative Ansätze zum möglichst schonenden chirurgischen Vorgehen etabliert. Die zunehmende Entwicklung minimalinvasiver Konzepte zeigt sich auch hier richtungsweisend mit einer Verkleinerung des operativen Zugangs unter Verwendung neuronavigationsunterstützter, stereotaktischer oder ultraschallgesteuerter Medien. Zusätzlich ergeben sich durch die Verwendung weiterer Therapieansätze, wie z. B. der hyperbaren Oxygenierung, neue fachübergreifende Möglichkeiten zur kurativen Therapie.

Neben der Verfeinerung neurochirurgischer Operationsmethoden sowie der Weiterentwicklung der Antibiotikatherapie erfordert die Behandlung des Hirnabszesses weiterhin größtmögliche Sorgfalt und Erfahrung, da die Mortalitätsraten in der Literatur weiter zwischen 9 und $33 \%$ angegeben werden. Weil nach neuesten mikrobiologischen Studien Hirnabszesse zu 46-89\% von anaeroben Keimen verursacht werden, wurde in den letzten Jahren die hyperbare Sauerstofftherapie als zusätzliches Instrument zur Behandlung herangezogen. Hierdurch werden Verringerung des perifokalen Ödems und des Hirndrucks sowie eine intermittierende Öffnung der
Blut-Hirn-Schranke mit erleichtertem Eintreten von Antibiotika in die Abszesshöhle erreicht. Weitere synergistische Effekte mit Antibiotika sind bekannt, zusätzlich besteht eine direkte Toxizität gegen anaerobe Keime.

\section{Patientengut und Behandlungsstrategie}

In den Jahren 1999-2001 wurden $11 \mathrm{~Pa}$ tienten nach neurochirurgischen Eingriffen bei Hirnabszessen mit hyperbarer Oxygenierung behandelt, hierunter waren 8 Männer und 3 Frauen, die Altersspanne betrug 16-89 Jahre. Als Infektionswege konnten 4 Infekte per continuitatem nachgewiesen werden, eine hämatogene Ursache war bei 5 Patienten nachweisbar, iatrogene Infekte nach neurochirurgischen Eingriffen traten in 2 Fällen auf. Bei allen Patienten wurden neuronavigations- oder ultraschallunterstützt offene Minikraniotomien oder Bohrlochkraniotomien durchgeführt (Abb. 2). In 6 Fällen konnten Anaerobier isoliert werden, bei einer Patientin war Aspergillus nachzuweisen. Die beiden iatrogenen Infekte waren durch Staph. aureus verursacht. An Antibiotika wur-

\footnotetext{
(c) Springer-Verlag 2003

Dr. Marc Schaan

Neurochirurgie, BG-Unfallklinik Murnau, Prof.Küntscher-Straße 8, 82418 Murnau, E-Mail:mschaan@bgu-murnau.de
} 
Trauma Berufskrankh 2003 - 5 [Suppl 2]: S305-S308 DOI 10.1007/s10039-002-0648-1

M. Schaan · M. Bierschneider · A. Kemmer · H. Jaksche

\section{The treatment of brain abscess: an interdisciplinary challenge}

\section{Abstract}

From 1999-2001, 11 patients with brain abscess were treated neurosurgically and with hyperbaric oxygenation (HBO): 8 men, 3 women in the age of $16-89$ years. The pathogenesis was per continuitatem in 4 cases, haematogenous spread in 5 cases, postoperative in 2 cases. All patients underwent neuronavigation or ultrasound guided surgical intervention. Anaerobic pathogens were insolated in 8 patients, Aspergillus in 1 case, 2 postoperative infections with Staph. aureus were registered. All patients underwent at least 10 treatment sessions of HBO (2,4 bar for $140 \mathrm{~min}$.) One clinically inapparent infarction close to the abscess cavity was seen in routine CCT, the follow up CCT showed a complete regression of the infarct. One patient died. There was no abscess recurrence in follow up of 8 months. HBO-therapy provides the neurosurgeon with an additional tool in treating brain abscess caused by anaeobic pathogens. However, final conclusions regarding clinical outcome can only be drawn after clinical experience with a larger patient collective.

\section{Keywords}

Brain abscess - Neuronavigation · Hyperbaric oxygenation · Anaeobics

\section{Septische Chirurgie}

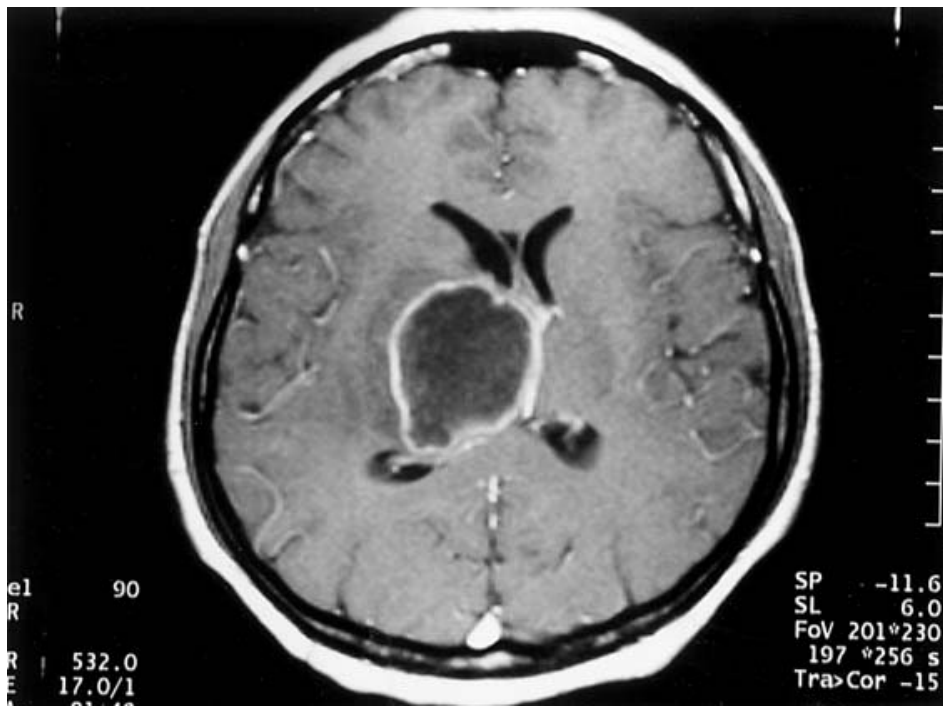

Abb. $1 \Delta$ Rechts im Stammganglienbereich gelegener Abszess im Stadium der späten Kapselentwicklung

den Cephalosporine der 3. Generation, Aminoglykoside, Zyvoxxid, Fosfomycin sowie Chloramphenicol und Metronidazol angewendet. Kortikosteriode kamen bei klinisch apparentem perifokalem Ödem mit deutlicher Raumforderung im CCT zur Anwendung.

Alle Patienten wurden mit mindestens 10 Behandlungssitzungen nach dem Problemwundenschema (2,4 Bar über 140 min.) in unserem Zentrum für hyperbare Medizin behandelt, die Behandlungsdauer wurde individuell anhand des klinischen Bildes, CCT-Befundes sowie der infektiologischen Parameter im interdisziplinären Konsens bestimmt.

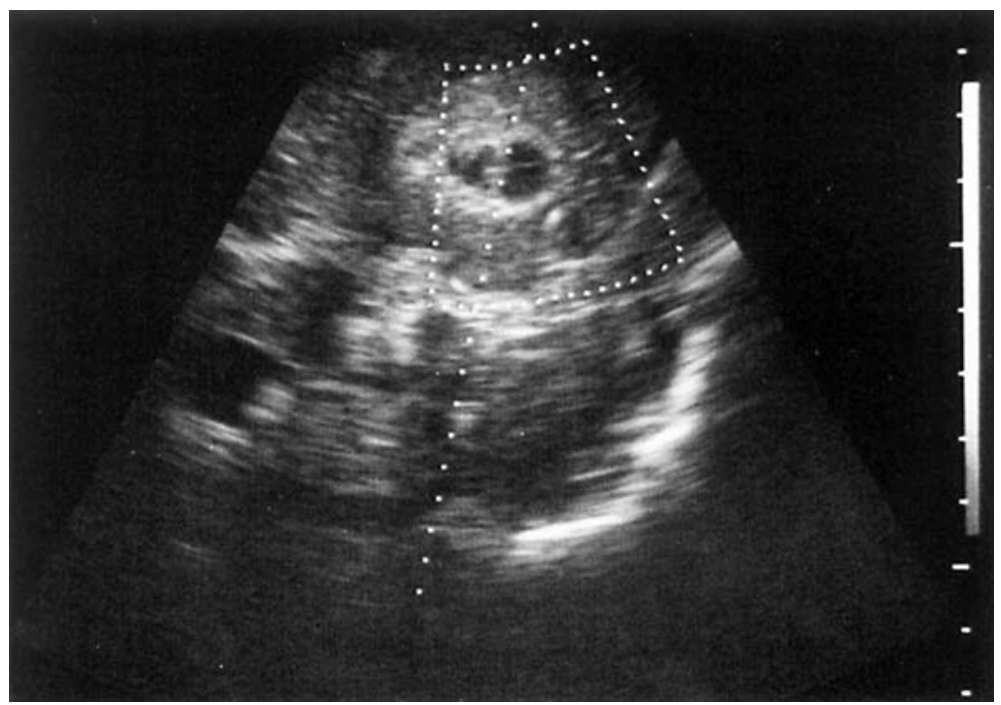

Abb. $2 \Delta$ Ultraschallgesteuerte intraoperative Darstellung eines Abszesses mit Planung der Punktionsrichtung 


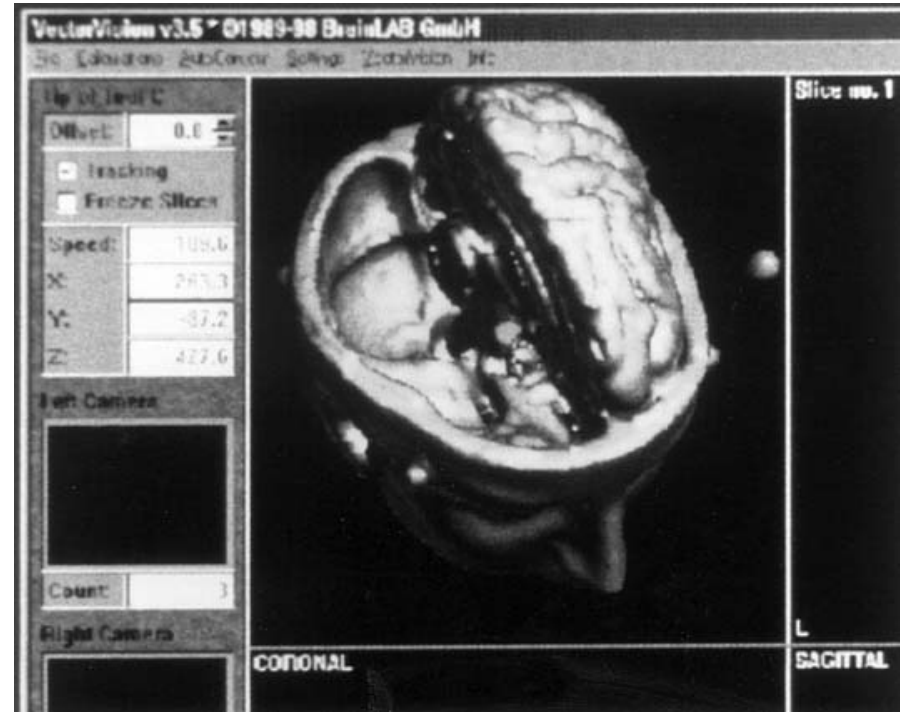

Abb. $3 \triangle$ Dreidimensionale Rekonstruktion anhand der Computertomographie mittels Neuronavigation

bedingten Fällen lagen singuläre Hirnabszesse vor.

In dem Fall eines 46-jährigen Patienten mit hämatogenem Abszess rechts parietal bedingt durch Bacterioides capillosus trat im Verlauf der HBO-Therapie ein klinisch inapparenter Infarkt in der Umgebung der Abszesshöhle auf. Die HBO-Therapie wurde nach 10 Sitzungen beendet, der Infarkt zeigte sich in den Verlaufskontrollen des CCT komplett regredient, eine neurologisches Defizit verblieb nicht.

Eine 55-jährige Patientin verstarb an einer therapeutisch nicht beherrschbaren disseminierten zerebralen Aspergillusaussaat bedingt durch eine Otitis media.

\section{Diskussion}

Trotz zunehmender Therapieverbesserungen stellt der Hirnabszess weiterhin eine neurochirurgische Erkrankung mit hoher Mortalität dar, diese wird in der Literatur mit 9-33\% angegeben [2]. Weiterhin besteht eine signifikante Morbidität. Die Inzidenz liegt bei 2-3 Erkrankungen pro 1 Mio. Einwohner.

\section{Pathogenese des Hirnabszesses}

Als Infektionsrouten sind hämatogene Streuungen (hauptsächlich bei Lungenabszessen, Empyemen, Bronchiektasen, arteriovenösen Lungenfisteln, kongenitale Herzfehler bei Kindern sowie bak- terielle Endokarditiden), eine Ausbreitung per continuitatem (z. B. otogen oder rhinogen), Infektionen der Kopfweichteile und posttraumatische Genesen bekannt.

Die Lokalisation der Hirnabszesse ist von verschiedenen pathogenetischen Faktoren abhängig, neben der Infektionsroute spielen auch Alter und Geschlecht des Patienten eine Rolle. Posttraumatische Hirnabszesse finden sich gehäuft im Frontallappen entsprechend des vorherrschenden Verletzungsmodus, bei kardiogenen Hirnabszessen ist be- vorzugt das Versorgungsgebiet der A.cerebri media betroffen. Otogene Abszesse zeigen häufig eine Beteiligung der mittleren oder hinteren Schädelgrube.

Das Keimspektrum umfasst in 46$89 \%$ der Fälle anaerobe Keime, die in der Vergangenheit sich darstellende hohe Anzahl steriler Kulturen wird im Allgemeinen auf die Güte der verwendeten Kulturtechnik zurückgeführt [4]. Die histologische Entwicklung eines Hirnabszesses wird in 4 Phasen unterteilt [1]:

- Zu Beginn lässt sich ein Stadium der nur schwer abzugrenzenden phlegmonösen Entzündung finden. Die Dauer dieses Stadiums liegt bei 13 Tagen.

- Das nachfolgende Stadium der späten Zerebritis ist durch eine zunehmende Nekrose und Ödementwicklung gekennzeichnet und dauert bis zu 2 Wochen.

- In der anschließenden frühen Kapselentwicklung (2-4 Wochen) zeigt sich eine beginnende Neovaskularisation mit Nekrotisierung.

- Die letzte Phase der späten Kapselentwicklung zeigt ebenfalls das klassische computertomographische Bild mit ringförmiger Kontrastmittelanreicherung, wobei sich hier intraoperativ die Kapsel als von zunehmend derber Struktur mit teilweise nur schwerer Penetrationsmöglichkeit darstellt.

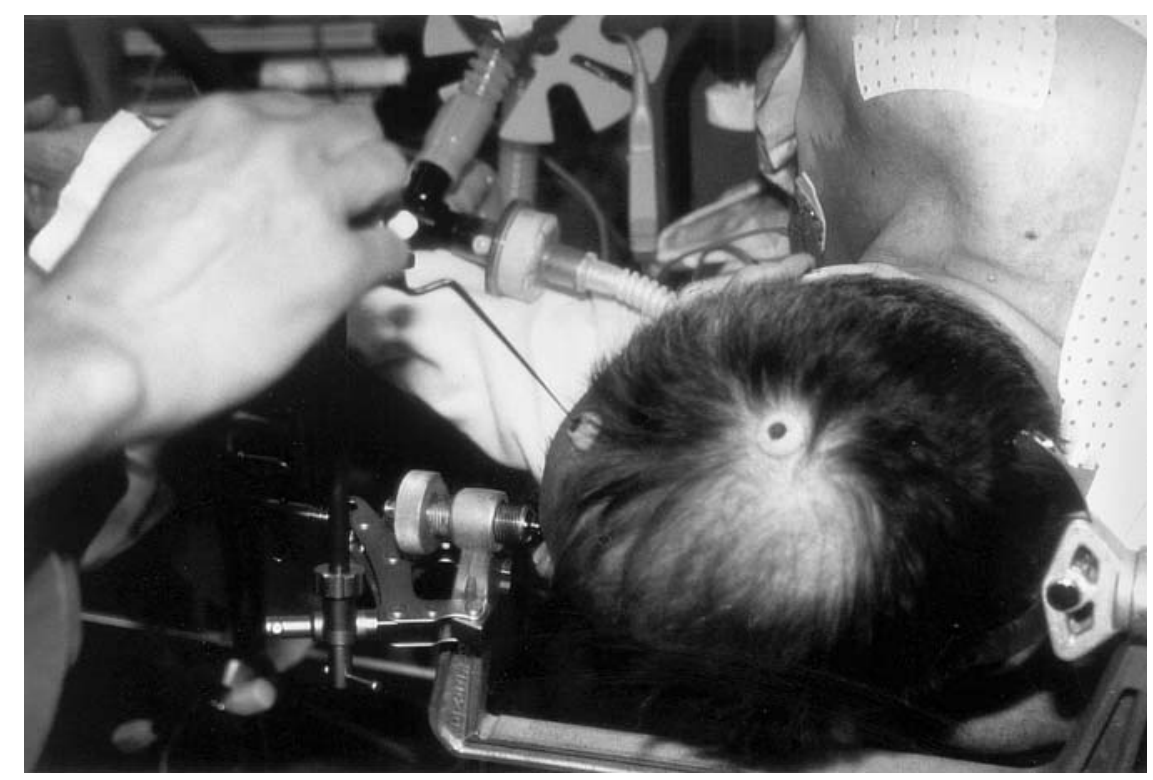

Abb. $4 \Delta$ Intraoperative Lokalisationsdiagnostik mittels Neuronavigation 


\section{Septische Chirurgie}

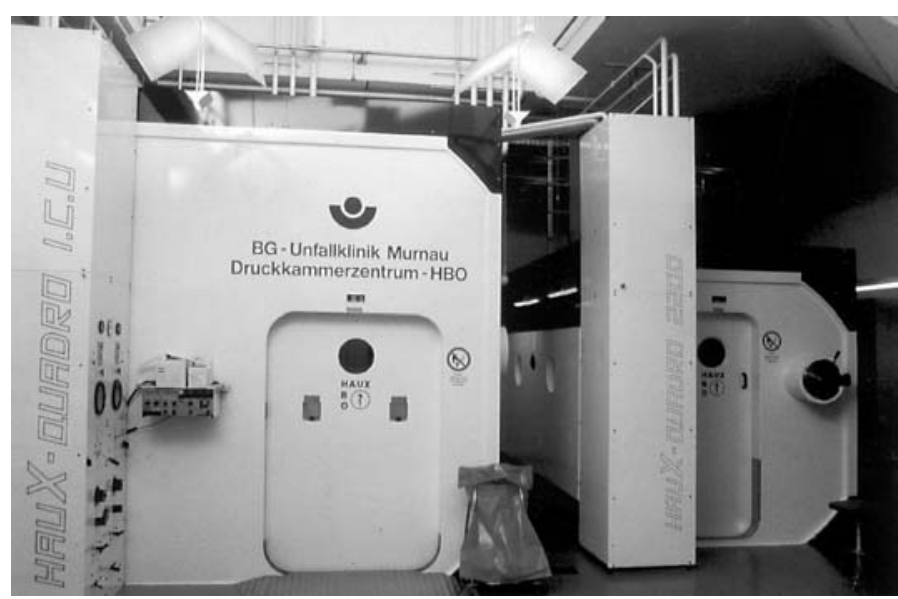

Abb. $5 \Delta$ Das Druckkammerzentrum der BG-Unfallklinik Murnau

\section{Verfeinerte neurochirurgische Methoden}

Die Indikation zum operativen Vorgehen wird anhand des klinischen Bildes, Lage und Größe der Raumforderung sowie der histologischen Entwicklungsstufe bestimmt. Neben einer möglichst frühzeitigen Diagnosestellung mit konsekutiver Therapiezuführung zeigt sich eine erfolgreiche Therapie des Hirnabszesses in der zunehmenden Verfeinerung operativer Techniken unter der Verwendung neuer apparativer Möglichkeiten sowie zusätzlicher Therapieoptionen. Stereotaktische, computertomographisch unterstützte Abszesspunktionen haben den Vorteil, dass über eine komplikationsarme Bohrlochtrepanation der Abszess punktiert und auch Drainagekatheter eingebracht werden können. Häufig können solche Eingriffe auch in Lokalanästhesie durchgeführt werden [3].

Mittels der neuronavigationsunterstützten Operationsmethode [5] ist es ebenfalls möglich, über eine Bohrlochkraniotomie oder ein erweitertes Bohrloch exakt eine intrazerebrale Raumforderung zu lokalisieren und mit minimal invasivem Aufwand eine operative Entlastung unter möglichst optimaler Schonung des umliegenden gesunden Hirngewebes durchzuführen. Hier zeigt die präoperative dreidimensionale Planungsstruktur eine besonders gute Vorbereitungsmöglichkeit für den Eingriff (Abb. 3, 4).

Auch zeigte die zunehmende Verwendung der intraoperativen Ultraschalldiagnostik eine deutliche Verringerung des operativen Traumas [5]. Mit Hilfe dieses Mediums ist es möglich, exakt die Relation von Hirnabszess und operativem Zugang bzw. zu einer Punktionskanüle $\mathrm{zu}$ ermitteln, auch können durch die simultane Verwendung von Duplexsonographie und Ultraschall während der Operation evtl. zu verletzende Gefäße identifiziert und geschont werden.

\section{Effekte der hyperbaren 0xygenierung}

Ein schwerwiegendes Problem der Behandlung intrakranieller Abszesse ist das Erreichen therapeutischer Antibiotikaspiegel in der Abszesshöhle. Dies ist in erster Linie bedingt durch die eingeschränkte Permeabilität der Blut-HirnSchranke und der Abszessmembran. Diese Probleme auch unter Berücksichtigung des vorherrschenden Keimspektrums stellen hervorragende Argumente für die Anwendung von hyperbaren Oxygenierung (HBO) in der Behandlung solcher Patienten dar (Abb. 5).

Neben der experimentellen Anwendung bei Schädel-Hirn-Traumen, Hirnblutungen und zerebralen Infarkten ist die Anwendung der HBO-Therapie bei Hirnabszessen generell anerkannt. Der zugrundeliegende Effekt ist hier die therapeutische Anwendung des Medikamentes Sauerstoff unter hyperbaren Bedingungen. Hierdurch lassen sich als physiologische Effekte eine direkte Toxizität auf anaerobe Keime, verbesserte Mikrozirkulation, Erhöhung der Leukozyten- und Makrophagenaktivität, verbesserte Mikrozirkulation sowie eine Reduktion von Hirnödem und Hirndruck durch Vasokonstriktion erreichen. Unter hyperbaren Bedingungen wird durch eine verstärkte physikalische Lö- sung im Plasma neben der Hämoglobinbindung eine übermaximale Sauerstoffsättigung des Gewebes mit oben genannten therapeutischen Effekten erreicht. Zusätzlich wird neben der Öffnung der Blut-Hirn-Schranke synergistisch eine Verstärkung der Antibiotikawirkung insbesondere bei Verwendung von Aminoglykosiden erzielt [6].

In Anbetracht der niedrigen Inzidenz von Hirnabszessen lässt sich eine statistisch signifikante Aussage bezüglich des Nutzens der HBO-Therapie noch nicht treffen, hier werden in der Literatur hauptsächlich Einzelfälle oder niedrige Fallzahlen beschrieben. Die jedoch bisher dokumentierten Verläufe zeigen ebenso wie unser hier vorgestelltes Kollektiv eine deutliche Verbesserung von Mortalität und Morbidität.

\section{Fazit für die Praxis}

1. Durch die Verwendung neuer technischer supportiver Methoden lässt sich eine deutliche Verringerung des operativen Traumas mit Senkung des operativen Risikos erreichen. Hierdurch lässt sich eine schonendere kurative Therapie erreichen.

2. Die Anwendung der hyperbaren 0xygenierung im interdisziplinären Kontext führt zu einer Verbesserung von Mortalität und Morbidität. Statistisch signifikante Aussagen können erst nach Untersuchung eines größeren Patientenkollektivs getroffen werden.

\section{Literatur}

1. Britt RH, Enzmann DR, Remington JS (1983) Clinical stages of human brain abscesses on serial CT-scans after contrast infusion.J Neursurg 59: 972

2. Colli BO, Carlotti CG, Machado HR, Assirati JA (1999) Intracranial bacterial infections. Neurosug Quart 9: 258-284

3. Hollander D, Villemure JG, Leblanc R (1987) Thalamic abscess: a stereotactically treatable lesion. Appl Neurophys 50: 168-171

4. Jamjoom $A B$ (1996) Short course antimicrobial therapy in intracranial abscess. Acta Neurochir 138: 835-839

5. Koivukangas J, Louksisalmi MS, Alakuijala J, Oikarinen J (1993) Ultrasound-controlled neuronavigator-guided brain surgery. J Neurosurg 79: 36-42

6. Lampl LA, Frey G, Dietze T, Trauschel M (1989) Hyperbaric oxygen in intracranial abscesses. J Hyperb Med 4: 111-125 